\title{
Théorie de la fiction et esthétique des jeux
}

\section{Olivier Caïra}

\section{OpenEdition}

\section{Journals}

Édition électronique

URL : http://journals.openedition.org/sdj/671

DOI : $10.4000 /$ sdj.671

ISSN : 2269-2657

\section{Éditeur}

Laboratoire EXPERICE - Centre de Recherche Interuniversitaire Expérience Ressources Culturelles Education

Référence électronique

Olivier Caïra, "Théorie de la fiction et esthétique des jeux », Sciences du jeu [En ligne], 6 | 2016, mis en ligne le 12 octobre 2016, consulté le 19 avril 2019. URL : http://journals.openedition.org/sdj/671 ; DOI : $10.4000 /$ sdj.671

Ce document a été généré automatiquement le 19 avril 2019.

Tous droits réservés 


\title{
Théorie de la fiction et esthétique des jeux
}

\author{
Olivier Caïra
}

1 L'appréciation esthétique d'un jeu semble au premier abord peu problématique. Nous savons tous apprécier la facture d'un échiquier, les illustrations d'un jeu de plateau, le rendu graphique ou la musique d'un jeu vidéo. Tous les amateurs de jeux reconnaissent l'apport à leur loisir d'artistes divers (illustrateurs, maquettistes, sculpteurs, scénaristes, acteurs, musiciens), et des expositions comme "L'art dans le jeu vidéo " (Paris, Art Ludique, septembre 2015-mars 2016) valorisent cette dimension, au moins pour le jeu numérique.

2 Le propos de cet article n'est pas d'insister sur les contributions des arts au jeu, mais d'étudier ses aspects esthétiques sous sa forme la moins ornementée. Pourquoi qualifionsnous un problème d'échecs de "splendide », un système de règles d'« élégant ", une énigme de jeu vidéo de "subtile », etc. ? Je partirai de la théorie que j’ai développée dans Définir la fiction (Caïra, 2011) pour examiner les appuis esthétiques proprement ludiques que l'on découvre dans les jeux abstraits et dans les jeux de simulation.

\section{Le jeu au cœur de la fiction}

3 Définie dès les premiers temps de la ludologie comme fictive et séparée du quotidien, l'expérience ludique est longtemps restée ignorée des théoriciens de la fiction. Il a fallu les textes pionniers de Jean-Marie Schaeffer (1999) et de Marie-Laure Ryan (2001, 2006) pour que le jeu vidéo entre dans la discussion, non comme épiphénomène mais comme manifestation de la demande contemporaine de fictions interactives. Comme l'indique son sous-titre «Du roman au jeu d'échecs ", Définir la fiction poursuit et théorise cet élargissement, plaçant les jeux au cœur de la palette des fictions. Deux aspects de cette théorie seront utiles ici : d'une part l'idée que la fictionnalité n'est pas une propriété des êtres ou des œuvres, mais le fruit d'un cadrage; d'autre part l'affirmation que les jeux abstraits forment un pôle de la création fictionnelle, distinct et parfois complémentaire des œuvres mimétiques. 


\section{Une définition pragmatique de la fiction}

L'ouvrage part de l'écart entre les objets examinés par les théoriciens - majoritairement des romans classiques - et l'éventail de ce que nos contemporains citent comme des œuvres ou expériences de fiction : films, jeux divers, sketchs, exercices mathématiques de «robinets» ou de "trains qui se croisent ${ }^{1}$ », etc. Toutes les fictions ne sont pas linguistiques, ni narratives (on parle d'ailleurs de plus en plus de "gradients de narrativité ", notamment pour les jeux de rôle et les jeux vidéo, cf. notamment Baroni et Marti, 2014), ni même mimétiques: des jeux formels comme le Go ou Tetris peuvent également être cités lors des enquêtes. Il est donc difficile de définir la fiction sur une base ontologique ou sémantique, les êtres qui la "peuplent» pouvant être des personnages historiques, des êtres imaginaires, voire de pures abstractions. La fiction ne se distingue pas de la réalité, elle l'enrichit.

5 La frontière pertinente passe entre fiction et documentaire : c'est ainsi qu'on organise une bibliothèque ou qu'on segmente le programme d'une chaîne de télévision. Non parce qu'il y aurait un «langage de la fiction » distinct de l'expression documentaire, mais parce que les contraintes pragmatiques qui pèsent sur cette dernière sont en partie levées. La fiction n'est pas un phénomène sémantique ou stylistique, mais le résultat d'un cadrage, au sens de la sociologie d'Erving Goffman (1991), qui lève la contrainte de preuve sur la communication. La ressemblance avec des personnages, objets ou faits réels est possible, mais elle serait, comme l'écrivent les studios hollywoodiens dans les génériques de films, «purement fortuite ».

6 Si la mimésis n'est plus une caractéristique nécessaire de l'expression fictionnelle, les critères de l'appréciation ne se limitent plus à la question de la représentation. On redécouvre ainsi toute une tradition d'étude des systèmes formels, qui n'a jamais écarté la question esthétique, depuis les écrits mathématiques de Platon et Aristote jusqu'à des auteurs contemporains (Hofstadter, 2000 ; Cléro, 2004). La théorie des cadres permet par ailleurs d'étudier aussi bien les œuvres que les expériences de fiction, ce qui incite à rechercher les aspects esthétiques des jeux en tant que produits culturels et en tant que supports d'un vécu sans équivalent dans le quotidien. On étudiera ainsi, plus loin, l'interaction entre "problémistes» et «solutionnistes» dans la pratique des échecs artistiques et dans les jeux d'énigmes (puzzle games), suivant une dynamique d'expérience ludique et non comme corpus figé d'énigmes (je reprends en cela la dynamique «riddler/ riddlee » proposée pour l'étude des énigmes dans Hasan-Rokem et Shulman, 1996).

\section{Pôles mimétique et axiomatique}

7 Cette définition de la fictionnalité a un impact majeur en termes d'analyse des jeux. Elle invite à réexaminer la palette des objets traditionnellement étudiés par les théoriciens. $\mathrm{Au}$ lieu d'évacuer les fictions logico-mathématiques telles que les Dames, le Backgammon ou Tetris (Pajitnov, 1984) comme des "cas à part ", trop éloignés du modèle de la représentation, l'approche pragmatique permet de réconcilier les traditions de la fiction mimétique et de la fiction axiomatique.

8 En effet, s'il a été possible jusqu'au $\mathrm{XX}^{\mathrm{e}}$ siècle de penser séparément les fictions mimétiques (roman, film ou sketch) et axiomatiques (jeux abstraits, systèmes logiques, géométries non-euclidiennes, etc.), l'émergence des jeux de simulation à partir des 
années 1970 invite à étudier les articulations entre ces deux catégories. Développer un jeu de simulation, c'est associer un thème à un système: d'une part la représentation d'une diégèse, de personnages, de péripéties, d'autre part un moteur logico-mathématique intégrant les choix des joueurs et produisant des résultats en accord avec le thème. Cette dualité peut être assumée par un seul créateur, comme c'est le cas dans les jeux de société jusqu'à la présentation des prototypes, mais elle nécessite le plus souvent un dialogue entre métiers créatifs, dont les positions peuvent également figurer sur l'axe du mimétique à l'axiomatique, de l'illustration au level design.

Le jeu de simulation apparait en position centrale dans la palette de Définir la fiction. Celleci forme un continuum : les frontières entre fiction mimétique et jeu de simulation sont de plus en plus floues (en témoignent les productions de Telltale Games, au confluent du jeu vidéo et de la série télévisée), et bien malin qui saurait tracer une limite claire entre jeux thématisés et jeux abstraits (les Échecs peuvent être vus tantôt comme une simulation militaire, tantôt comme la quintessence du jeu sans référent). Roger Caillois évoque cette dualité quand il pose le caractère fictif du jeu : «conscience spécifique de réalité seconde ou franche irréalité par rapport à la vie quotidienne " (Caillois, 1967, p. 42). Cette palette élargie et rééquilibrée des fictions permet de poser la question de l'esthétique sans préjugés, sans chercher à " plaquer » les concepts de l'esthétique de la représentation sur les jeux abstraits ou sur le volet « système » des jeux de simulation.

\section{Sublime, subtil et merveilleux dans les jeux abstraits}

10 Un jeu abstrait n'a d'intérêt que s'il nous pose un défi intellectuel. Un système entièrement analysé n'a plus d'attrait : on ne peut que le ressasser, le parcourir, sans plus rien y découvrir. C'est le cas du Marienbad ou du Tic-Tac-Toe. Au Marienbad, la position de départ est perdante : quoi que fasse le premier joueur, on peut toujours le replacer en position perdante, jusqu'à la défaite. Dès lors que les deux joueurs connaissent son déploiement, le Marienbad n'est plus un jeu, mais une axiomatique «démolie ». Au TicTac-Toe, l'issue est systématiquement la partie nulle.

11 À l'opposé, la prise de conscience de nos limites, de notre échec à embrasser par la pensée tous les coups d'un jeu, conduit à une expérience particulière, celle du sublime. L'exploration du Go ou des Échecs engendre une dialectique esthétique : repousser les limites de notre connaissance renvoie à notre finitude, nous rend humbles face à l'immensité de l'inexploré, mais procure le plaisir d'avoir progressé malgré cette condition.

12 Cette conception est longtemps demeurée, du fait de l'influence de Kant, l'axe de réflexion dominant sur l'esthétique des jeux abstraits :

Le sentiment du sublime est ainsi un sentiment de peine, suscité par l'insuffisance

de l'imagination dans l'évaluation esthétique de la grandeur pour l'évaluation par

la raison; et en même temps il se trouve en ceci une joie, éveillée justement par l'accord entre les Idées rationnelles et ce jugement sur l'insuffisance de la plus puissante faculté sensible, dans la mesure où c'est pour nous une loi que l'effort de tendre vers ces Idées (Kant, 1989, p. 96).

Cependant, l'esthétique dans une fiction axiomatique ne se résume pas au sublime. Il peut aussi y avoir subtilité dans un problème d'Échecs, une position de Ricochet Robots (Randolph, 1999), un niveau de Portal (Valve Software, 2007). Nous étudierons aussi le merveilleux, fondé la découverte des propriétés d'un système de règles. 


\section{Formalisme et hédonisme : les Échecs artistiques}

C'est un art magnifique, complexe et stérile, parent de la forme ordinaire du jeu seulement dans la mesure où, par exemple, le jongleur, en inventant les entrelacements d'un nouveau tour d'adresse, et le joueur de tennis, en gagnant un tournoi, tirent tous deux partis des propriétés d'une sphère. En fait, la plupart des joueurs d'échecs, les maîtres comme les amateurs, ne prennent qu'un intérêt modéré à ces énigmes raffinées, de pure fantaisie et relevant d'une grande spécialisation et, bien qu'ils apprécient un problème renfermant un piège, ils seraient absolument déconcertés si on leur demandait d'en composer un (Nabokov, 1991, p. 364).

Vladimir Nabokov décrit en praticien la spécificité des échecs artistiques, cet usage du jeu qui consiste à composer des problèmes en dehors du cadre ordinaire de la confrontation entre deux joueurs. Les amateurs d'échecs artistiques ne sont pas des adversaires départagés par un mat, mais des "problémistes » (ou " compositeurs ») qui publient des énigmes pour des « solutionnistes » qui tentent de les résoudre.

L'art s'y exprime en deux sens: une discipline fondée sur la régularité et l'approfondissement, proche d'un artisanat, et une dynamique créative proche des beauxarts. En marge de la compétition échiquéenne, le monde des problémistes s'est doté de règles de création et de systèmes d'évaluation qui vont nous guider vers d'autres jeux abstraits. Les normes techniques de la discipline se divisent en deux catégories : les règles absolues et les règles relatives (Lautier, 2002, p. 304-305).

\begin{tabular}{|c|c|}
\hline Règles absolues & Règles relatives \\
\hline $\begin{array}{l}\text { La position doit être légale, c'est-à-dire qu'elle doit respecter les règles } \\
\text { du jeu d'échecs. } \\
\text { Les blancs jouent et font mat en «n » coups et cela quelles que soient } \\
\text { les défenses envisagées par les noirs (si une solution plus rapide est } \\
\text { trouvée, alors le problème est « démoli »; si une défense inattaquable } \\
\text { apparait, alors le problème est dit insoluble). } \\
\text { Le premier coup - la « clé »- est unique. }\end{array}$ & $\begin{array}{l}\text { Pas de clé d'échecs. } \\
\text { Pas de clé commençant } \\
\text { par une prise. } \\
\text { Pas de clé équivalant à } \\
\text { une promotion en } \\
\text { Dame. } \\
\text { Pas de clé synonyme } \\
\text { de prise d'une case de } \\
\text { fuite du Roi. }\end{array}$ \\
\hline
\end{tabular}

Les premières règles de chacune des catégories définissent les échecs artistiques : elles instituent la discipline en lieu et place des règles de la compétition échiquéenne. Les secondes permettent de distinguer une «bonne » composition d'un problème ordinaire, position intéressante survenue au cours d'une compétition et relevée par un spécialiste. L'immense majorité de ces dernières se résout par l'un des quatre moyens que les règles relatives proscrivent. C'est parce que la dimension stratégique de la partie est remplacée 
par l'intentionnalité de la composition que les échecs artistiques engendrent des positions inusitées.

Viennent ensuite des règles « esthétiques » :

- Il faut respecter le principe d'économie des forces et des moyens, c'est-à-dire placer un nombre minimum de pièces sur l'échiquier et construire une solution à la fois unique et belle.

- Il est conseillé d'utiliser un nombre réduit de pièces essentielles et d'éviter le plus possible d'avoir recours aux "chevilles ", c'est-à-dire aux pièces qui servent uniquement à la construction du problème et qui restent passives.

- La multiplication des thèmes est un facteur qualitatif important qui ne doit pas se faire au détriment d'une certaine harmonie.

- L'originalité d'un problème est indispensable, comme dans toute création.

- À partir de la position donnée, il existe un ou plusieurs coups évidents qui ne sont bien sûr jamais la vraie clé mais qui vont la mettre en valeur, leur multiplicité apportant un plus à l'œuvre.

- Si possible, les différentes variantes répondant à l'énoncé doivent comporter le même nombre de coups et les coups blancs doivent être identiques.

- La difficulté du problème et de la clé est un facteur de qualité : la satisfaction du solutionniste est proportionnelle aux efforts qu'il aura consacrés à la résolution du problème (Lautier, 2002, p. 304-305).

On constate que les épithètes " technique " et " esthétique " n'instaurent pas un découpage strict entre l'agencement de la position et son appréciation. Toutes les règles des échecs artistiques sont indissociablement techniques et esthétiques, au sens où la beauté d'un problème est toujours une résultante de son élucidation. Voici un problème posé par Thomas Taverner dans le Dubuque Chess Journal (1889), commenté à la page " Problème d'échecs » de Wikipédia :

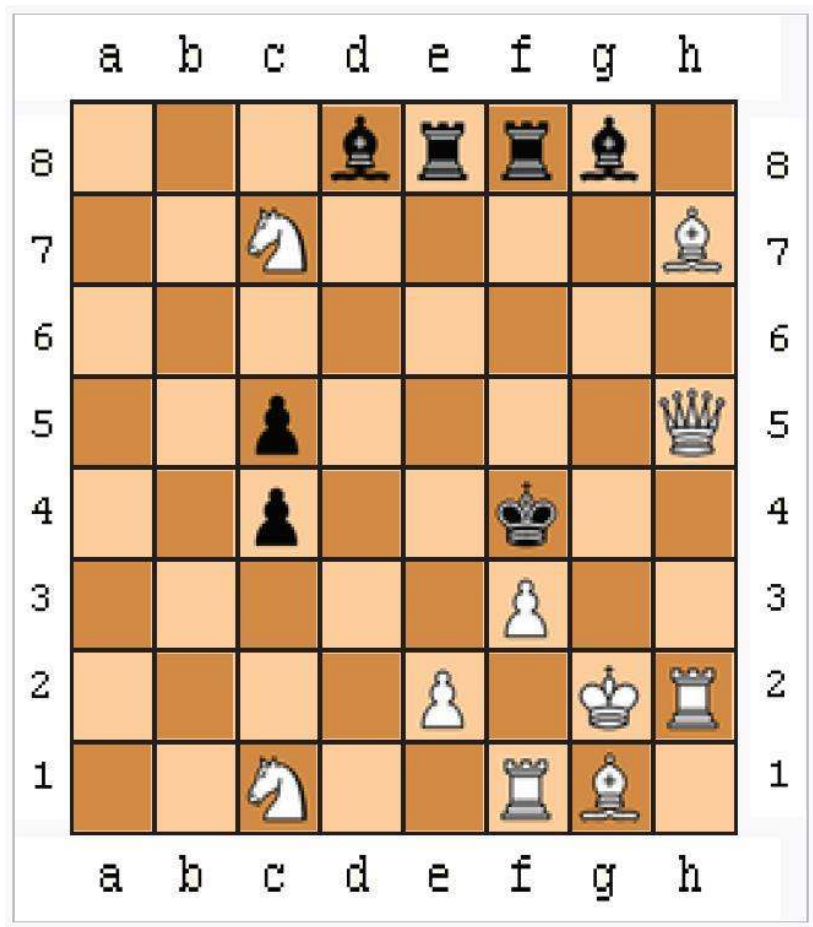

Les blancs jouent et matent en deux coups 

n'est clairement pas picturale. Sa beauté se révèle d'abord par l'élimination des coups les plus tentants. Au terme de cette exploration, la clé apparait enfin, un coup d'apparence anodine : Tour en h1. La subtilité tient à ce coup d'attente, qui respecte les quatre « règles relatives ": pas de capture ni de promotion d'un pion en dame, pas d'échec ni de prise d'une case de fuite du roi. Il illustre le motif du zugzwang, situation rarissime où chaque coup des noirs devient perdant alors que ne pas jouer leur aurait valu un répit. Taverner a bâti ce zugzwang en agençant les pièces noires afin que celles qui peuvent se déplacer (les Tours, les Fous et le Pion en c4) quittent une position de protection du Roi ou gênent les autres. C'est le motif secondaire du Grimshaw, la configuration « en tuyaux d'orgue » des Fous et des Tours qui se gênent mutuellement formant quatre Grimshaw simultanés.

L'expérience du subtil n'est donc pas celle du sublime. Elle émerge ponctuellement de l'engagement du joueur, qui surmonte une difficulté intellectuelle produite par un autre. Le problémistes ne saurait être décrit comme "génial» au sens de Kant, c'est-à-dire comme doté d'une créativité essentiellement inconsciente et échappant à toute codification. Comme tout game designer (et le terme design l'exprime bien), le problémiste ne produit d'effet esthétique qu'au moment où son œuvre est activement comprise, voire partagée, par son public, comme l'explique bien Chéron :

Ce que cherche le compositeur d'une fin de partie artistique, c'est à provoquer l'enthousiasme du chercheur par une combinaison extraordinaire, inattendue, brillante. CAR LA FIN DE PARTIE ARTISTIQUE N'EST AUTRE CHOSE QUE L'ART DES BELLES COMBINAISONS. [...] C'est un pendant naturel de l'esprit humain qui si une fin de partie artistique l'a enthousiasmé, il cherche à faire partager sa joie à son prochain (Chéron, 1971, p. 39).

\section{Des Échecs au jeu vidéo}

21 On identifie bien dans les Échecs artistiques une dimension proprement ludique de l'expérience esthétique à travers le défi intellectuel. Ce raisonnement peut être élargi. Toute énigme ludique est inséparable de son élucidation, elle tend à l'intelligibilité, mais laisse également ouvertes de nombreuses pistes, développe de nouvelles ramifications, pose des questions qui pointent également vers le sublime.

Les règles esthétiques des Échecs artistiques se retrouvent en filigrane dans de nombreux jeux d'énigmes qu'il s'agisse de jeux d'aspect purement numérique ou géométrique, le sudoku et ses nombreux avatars, les grilles de 2048 (Cirulli, 2014) ou SquareCells (Brown, 2015), les cubes mobiles de Edge (Mobigame, 2011), Rush (Two Tribes, 2010) ou Cubot (Pierre-Loti-Viaud, 2014), ou qu'ils dépeignent une diégèse naïve, abstraite ou absurde, comme le monde à plat de Fez (Polytron, 2012), les étranges boules de World of Goo (2D Boy, 2008), la déambulation grégaire des Lemmings (Psygnosis, 1991), etc. Dans les deux cas, on privilégie l'économie des éléments et la simplicité de leur représentation, ainsi que l'unicité de la solution. Si certains jeux d'énigmes autorisent plusieurs solutions, tout est fait pour valoriser la solution optimale : par exemple celle qui s'opère en un minimum de coups dans le jeu de plateau Ricochet Robots ou dans le jeu vidéo Cubot. Le temps peut être l'un des paramètres de l'énigme, mais il est souvent neutralisé en tant que mesure « externe » de la résolution.

23

L'attrait esthétique de ces jeux gît donc dans le dépouillement, à l'opposé de la conception qu'on a habituellement des jeux vidéo à budgets élevés, soit des environnements 
luxuriants développés en «bac à sable » comme les Grand Theft Auto ou The Elder Scrolls. Le slogan de Cubot l'annonce clairement: « La complexité de la simplicité » ("The Complexity of Simplicity »). Toute l'interface, depuis la police de caractères épurée jusqu'à la musique apaisante, est conçue pour favoriser la concentration. Les jeux d'énigmes peuvent même « s'appauvrir » au fil des éditions : Toki Tori (Two Tribes, 2001) est l'un des rares jeux qui, dans son deuxième volet, propose moins de fonctionnalités que dans le précédent: hormis les déplacements, le poussin de Toki Tori 2 (Two Tribes 2012) n'a que deux actions à sa disposition, contre une demi-douzaine dans le premier opus. Le jeu n'en devient pas plus simple: sa complexité réside désormais dans les créatures et mécanismes qui entourent le poussin.

\section{L'expérience du merveilleux}

24 Le mot «exploration » nous conduit vers une troisième forme d'expérience esthétique liée aux jeux abstraits: celle du merveilleux. Celle-ci traduit la découverte d'une propriété inattendue du système de règles. Elle ne survient pas au terme du processus de résolution, mais d'une recherche plus fondamentale, à mi-chemin entre l'utilisation « ordinaire » du jeu et le game design. Tandis que le subtil apparaît en suivant la logique déductive d'un problémiste, le merveilleux consiste à devenir son propre compositeur, par des raisonnements plus intuitifs et inductifs. C'est pourquoi les témoignages d'émerveillement vont surtout surgir à des tables de jeux classiques (Échecs, Go), mais aussi chez des utilisateurs assidus de jeux contemporains, découvrant par exemple une stratégie gagnante en apparence impossible dans des jeux de cartes comme Netrunner (Garfield, 1996), Dominion (Vaccarino, 2008) ou Race for the Galaxy (Lehmann, 2007), ou encore dans les éditeurs de scénarios et de niveaux (workshops) dédiés à des jeux vidéo comme Portal 2 (Valve, 2011) ou Toki Tori.

Si Portal 2 a pu décevoir certains critiques du fait de la simplicité de ses énigmes, les contributeurs de son workshop sur la plate-forme Steam l'enrichissent en expérimentant des combinaisons, au point que l'œuvre éditée apparaît aujourd'hui comme une sorte de didacticiel des "vraies» énigmes que l'on trouve en sommet de classement dans le workshop. Certains thèmes récurrents sont apparus autour des lasers, des cubes, des catapultes, des gels, des miroirs ou de la simple gravité, de la même manière que les problémistes d'échecs ont exploré les finales de pions, de tours ou de cavaliers pour en tirer des « familles » entières de compositions.

L'effet du merveilleux est d'autant plus stimulant que contre-intuitif : alors qu'on explore le système afin de mieux le maîtriser, on en découvre une propriété, une sorte de théorème, qui dévoile une ramification nouvelle de la combinatoire, un thème au sens où nous parlions plus haut du zugzwang aux Échecs. On ne découvre pas ce motif en quelques parties. De même, il faut jouer contre le bon sens défensif pour découvrir l'intérêt du backgame au Backgammon, puisque cette stratégie consiste à se faire capturer pour noyauter le camp adverse et retourner progressivement la partie en sa faveur. Le merveilleux ne se révèle que par une pratique assidue du jeu et de l'échange avec d'autres joueurs. Une fois compris, il peut donner lieu à un travail de problémiste.

Ces réflexions peuvent être rapprochées de la recherche en mathématiques, dans laquelle on retrouve fréquemment le terme « jeu ». En 1970, le Britannique John H. Conway, alors professeur à l'université de Cambridge créa l'une des plus célèbres axiomatiques autonomes de l'histoire des mathématiques: le Jeu de la vie. D'une extraordinaire 
simplicité, elle donne toujours lieu à des recherches approfondies, et sa postérité est considérable dans le domaine des automates cellulaires.

\section{Trois esthétiques en une figure : le problème de Smullyan}

Pour clore ce tour d'horizon sur les jeux abstraits, revenons aux Échecs artistiques, sous un de ses angles les moins connus : celui de l'analyse rétrograde. Ce sont des problèmes qui se jouent à rebours: il faut tantôt retrouver les trois derniers coups joués, tantôt déterminer si les Blancs ont encore le droit de roquer, ou indiquer sur quelle case une pièce a été prise. Le problème qui suit regroupe trois esthétiques en une figure : il est sublime par son ambition, subtil par son exécution et merveilleux par ses conséquences.

L'ambition de Raymond Smullyan, grand vulgarisateur de l'analyse rétrograde, est de trouver une position où « le trait étant aux Blancs, on peut prouver qu'ils peuvent jouer et faire mat en deux coups sans toutefois pouvoir exhiber le mat » (Smullyan, 1983, p. 86, texte et figure).

\section{NOIRS 3}

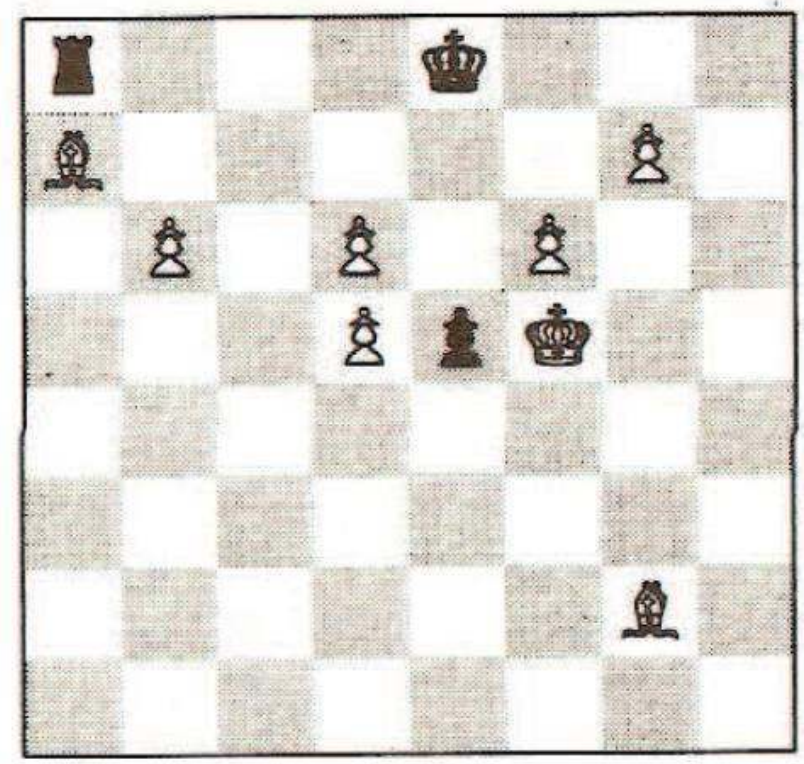

\section{BLANCS 8}

L'énoncé paraît classique : il tend vers le futur (la recherche du mat), non vers le passé. Puis tout se complique : on découvre deux scénarios pour mater en deux coups, mais chacun ne peut être exécuté qu'en sachant ce qui s'est produit auparavant. Si Noir n'a pas le droit de roquer, le Roi blanc vient en e6 et, quelle que soit la réponse de Noir, le Pion g7 mate ensuite par une promotion en h7. Si Noir a le droit de roquer, c'est qu'il n'a jamais déplacé ni sa Tour ni son Roi. Il a donc joué e7-e5 avec son Pion, ce qui autorise une prise en passant du Pion blanc ( $\mathrm{d} 5 \mathrm{xe}$ ), puis un mat par le pion de la colonne $b$ en cas de roque (la diagonale du fou s'étant libérée). On a bien démontré la possibilité du mat en deux coups sans pouvoir l'exhiber.

Exploitant les règles du roque et de la prise en passant, Smullyan dévoile une propriété du système, une sorte de théorème de Gödel des Échecs : l'existence de problèmes 
« indécidables». D'où l'expérience du sublime: notre connaissance de l'axiomatique progresse, mais nous n'en sommes que plus désarmés pour en comprendre toutes les circonvolutions, plus convaincus de l'immensité de ce qui reste à découvrir. D'où également l'expérience du subtil, car la composition d'un tel problème, limpide en apparence, nécessite une grande ingéniosité et invite à des efforts déductifs inédits. D’où enfin l'expérience du merveilleux, car c'est un nouveau genre de problèmes que Smullyan invente et découvre à la fois: une famille d'énigmes logiques et stratégiques, fonctionnant indissociablement, pour le problémiste comme pour le solutionniste, vers le futur et vers le passé.

\section{L'esthétique mixte des jeux de simulation}

Jeux de plateau, wargames, jeux de rôle, jeux vidéo, chacun de ces domaines éditoriaux a construit son propre espace d'évaluation, avec ses récompenses, sa presse et ses sites spécialisés, ses débats internes. Du fait de leur position centrale dans la palette des fictions définie plus haut, ces jeux présentent trois flancs pertinents pour l'esthétique :

- le flanc mimétique porte sur tout ce qui évoque le thème du jeu (invasion de zombies, spéculation immobilière, voyage dans le temps, gestion d'un zoo, etc.), tant par le rendu graphique, sonore voire tactile des systèmes de simulation que par des éléments additionnels au gameplay (jaquettes, cartes, boîtes de jeu, musique et effets sonores, figurines, cinématiques);

- le flanc axiomatique permet d'étudier la résolution des problèmes de conception et de programmation suivant les modalités du sublime, du subtil et du merveilleux ;

- le flanc que Gonzalo Frasca appelle « simiotique » (Frasca, 2001) consiste à étudier la relation entre le système de simulation et les phénomènes qu'il simule - entre le simulant et le simulé - comme élément irréductible aux deux premiers (on peut admirer les paysages d'un jeu vidéo, apprécier l'élégance de son code, et juger son système de simulation défectueux).

Concentrons-nous sur ce troisième aspect pour cerner la spécificité des expériences du beau liées à la simulation ludique. J'examinerai d'abord le cas des jeux de plateau et de rôle, qui doivent reposer sur un système de simulation assez simple pour être assimilé et géré par des humains. L'enjeu simiotique est donc de produire une règle épurée, très éloignée des phénomènes complexes qu'elle modélise. C'est bien entendu le contraire dans les jeux vidéo contemporains des grands studios, où le système de simulation n'est limité que par les moyens de développement et la puissance des machines.

\section{Jeux de cartes et de plateau : un art de l'épure}

Le mécanisme en « stop ou encore » est apparu dans des jeux abstraits, notamment sur les comptoirs des cafés avec le 10000. À mesure qu'on relance les dés croit le score... et le risque de tout perdre si on ne s'arrête pas à temps. C'est également le principe du Black Jack. Employé dans un jeu de simulation, ce type de système permet d'évoquer des thèmes du quotidien ou d'une vie plus aventureuse : le "stop ou encore" va donc servir de moteur à des dynamiques d'exploration souterraine comme Dragon Run (Cathala \& Ludtche, 2014), Diamant (Faidutti \& Moon, 2005), The Adventurers (Blossier \& Henry, 2009), d'escalade comme Can't Stop (Sackson, 1980) ou de plongée comme Abyss (Cathala \& Chevalier, 2014), autant de milieux dangereux dans lesquels savoir s'arrêter devient l'enjeu et la compétence centrale pour gagner. Les critiques de ces jeux portent souvent 
sur la «greffe », réussie ou non, du thème au système : l'effet esthétique de l'épure est d'autant plus marqué qu'un moteur rudimentaire en apparence produit des résultats similaires à ceux d'un environnement complexe.

Détaillons un exemple. Formule Dé (Lavaur \& Randall, 1991) propose de recréer la tension des courses de formule 1 par un double système: d'une part, le joueur peut déplacer à chaque tour son "levier de vitesse ", chaque vitesse correspondant à un dé numéroté en conséquence (première : la voiture avance de 1 ou 2 cases; seconde : dé numéroté de 2 à 4 cases, troisième : de 3 à $8 \ldots$ jusqu'à la sixième : de 21 à 30 cases); d'autre part, chaque virage comporte un nombre donné de cases et un nombre de tours à y passer. Ainsi, un virage comptant quatorze cases sur sa trajectoire la plus longue et imposant trois tours oblige les joueurs à rétrograder en troisième ou en seconde, faute de quoi leur voiture risque différents désagréments (tête-à-queue, carambolage, etc.).

À cette base ludique fort éloignée de la mécanique automobile viennent s'ajouter des points de règle obligatoires (gestion de la carrosserie, risque de caler au départ, etc.) ou facultatifs (gestion des conditions climatiques, choix des pneumatiques, essais chronométrés, etc.) qui permettent de raffiner les stratégies et de rapprocher la physionomie du jeu de celle d'une course. Cette fidélité a son prix : Formule Dé est ainsi l'un des rares jeux où l'on peut, comme dans un Grand Prix, perdre sa voiture dès les premiers tours de roue, dans les cafouillages du départ ou lors du premier freinage.

L'étonnement des joueurs naît de l'écart entre la simplicité de la règle et la complexité des sports mécaniques : comment peut-on prétendre simuler l'un par l'autre ? La réponse est double :

- par la physionomie des courses qui, bien que réduites à quelques tours, offrent l'essentiel des motifs (phénomènes d'accordéon entre courbes et lignes droites, différences entre circuits lents et rapides, arrêts massifs au stand en cas de pluie, casses inopinées, etc.) et passes d'armes (dépassements au freinage, jeux sur l'aspiration, gestion stratégique des arrêts, etc.) d'un Grand Prix ;

- d'autre part, par la palette des compétences travaillées et éprouvées par le jeu (gestion des probabilités, observation des configurations de course, utilisation raisonnée des points de résistance de la voiture jusqu'au drapeau à damier, etc.).

On peut donc affirmer que la greffe entre thème et système $a$ bien pris. Le succès d'un tel jeu n'est pas assimilable à celui d'un simulateur informatique dont la fidélité aux conditions de course reposerait sur la modélisation des trajectoires. Formule Dé séduit alors qu'il substitue à la conduite sportive un espace et un temps discrets (case par case, tour par tour) et qu'il remplace des hommes et des engins rodés durant des centaines d'heures par des voitures en plastique pilotées à l'aide de jets de dés. Le paradoxe n'est qu'apparent : la transparence du système permet d'exercer ses capacités de calcul et non ses réflexes; la vision du circuit en surplomb permet aussi bien de diriger ses bolides que de gérer son écurie ou d'évaluer la stratégie des concurrents. C'est un autre rapport à la course qui est proposé.

\section{Jeux de rôle : des règles pour un monde sans limites}

39 Les questions simiotiques dans les jeux de rôle sur table se posent de manière plus complexe qu'en jeu de plateau, pour au moins trois raisons. Ces jeux fonctionnent d'abord sur la base d'un renouvellement des parties par la scénarisation et par l'évolution des personnages, donc d'une diégèse largement indéterminée et évolutive. Ensuite, le simulé 
n'est pas seulement plus complexe que le simulant, il est sans limites : si un personnage lance du sable aux yeux de son adversaire dans un combat d'arène, ou renverse une pyramide de boîtes de conserve pour freiner ses poursuivants dans un hypermarché, il faudra trouver un moyen de simuler ces actions qui, bien entendu, ne figurent pas dans le manuel. Enfin, le système d'un jeu de rôle n'est pas prescriptif: il n'a ni vocation à désigner des gagnants ou des perdants, ni à simplifier les enjeux suivant une métrologie univoque de " points de victoire » ou de " coups gagnants».

Les joueurs affirment souvent que la première qualité d'une règle de simulation est de "savoir se faire oublier ", de ne pas créer d'anicroche ni de rupture dans l'engagement des joueurs. La règle peut perturber de plusieurs manières : en rallongeant la partie au point de lasser les joueurs (s'il faut des heures pour simuler une escarmouche), en engendrant des résultats invraisemblables (possibilité mathématique de survivre à une chute de mille mètres), ou encore en bafouant les exigences d'équité et de transparence (en accordant des pouvoirs exorbitants à une classe de personnages).

41 Cette complexité n'empêche pas de produire des discours esthétiques sur les règles. On y retrouve l'esthétique de l'épure, du système de jeu "simple et élégant », soit lorsque le système repose sur un mécanisme unique de résolution des actions, soit lorsqu'un point de règle simple permet ponctuellement de traiter un problème complexe. C'est le cas du fameux « plafond descendant » de Santé mentale dans l'Appel de Cthulhu (Petersen, 1981): à mesure que le personnage-joueur progresse dans ses compétences " Mythe de Cthulhu " croît le risque qu'il perde la raison, car son score maximal de santé mentale se calcule par «100 - Mythe de Cthulhu». On simule ainsi l'inexorable descente dans la folie des personnages lovecraftiens.

Analysons un exemple moins connu: le mécanisme de simulation de la dépendance dans C.O.P.S. (Siroz, 2003), qui propose de jouer des policiers de Los Angeles dans un futur proche où la Californie a fait sécession des États-Unis. Lignes blanches (2003), le deuxième supplément de règles et de contexte du jeu, aborde la double question de la surveillance des frontières et du trafic de stupéfiants. Dans la partie consacrée aux drogues, les auteurs proposent le système suivant :

[...] après chaque consommation, immédiatement après la fin de l'effet, le consommateur devra effectuer un jet de résistance basé sur sa plus petite caractéristique entre le Sang-Froid et la Carrure. Si ce jet est réussi, le consommateur s'en sort sans trop de dommage cette fois-ci, sinon il devient accro. Un jet de résistance à l'addiction devra être effectué après chaque prise de drogue. Si une même drogue est consommée à plusieurs reprises sur une période de 30 jours, chaque jet de résistance au-delà de la première prise verra sa difficulté augmenter de un de manière cumulative (+1 à la deuxième prise, +2 à la troisième...) (p.132).

La règle intègre ici les caractéristiques des substances (chacune possède un «degré d'addiction » chiffré) et des personnages (via leurs caractéristiques), en tenant compte du cumul des prises. Elle répond donc bien à l'indétermination générale de la diégèse : les personnages ne sont pas donnés mais créés, et de nouvelles drogues peuvent apparaître au fils des scénarios. De plus, faire porter l'épreuve sur la plus faible des deux caractéristiques du personnage reflète la tonalité sombre de l'univers de C.O.P.S. : on joue des policiers ordinaires et faillibles. Enfin, elle est conçue et "calibrée " pour avoir un effet dissuasif sur les choix des joueurs, sans toutefois apparaître comme un interdit :

La décision éventuelle d'un personnage de décider [sic] d'utiliser des drogues doit être laissée à l'appréciation de chaque joueur. Assurez-vous toutefois que vos 
joueurs comprennent bien le coût qu'un tel choix va représenter pour leur personnage. Nul ne sort indemne d'une consommation excessive d'alcool ou de tabac, et nous parlons ici de drogues aux effets cent ou même mille fois plus puissants et plus dévastateurs. N'hésitez pas à ruiner la vie d'un personnage décidant de s'engager sur un chemin aussi hasardeux, vous resterez de toute manière souvent bien loin de la vérité (p.131).

On voit que les thèmes réalistes et désespérés de C.O.P.S. peuvent s'appliquer de manière mathématique, sans restreindre la liberté des joueurs, car le système de simulation fonctionne ponctuellement comme un dispositif de sanctions et de récompenses.

Un autre pas est franchi lorsque la règle apparaît comme la matrice mythologique du monde. Pour rapprocher esthétiquement le simulant du simulé, la conception dépasse la simple construction d'un espace de probabilités: on élabore le mythe fondateur de l'univers à mesure qu'on en rédige les règles. Dans le jeu d'univers parallèles Everway (Tweet, 1995), les règles de création des personnages et de résolution des situations reposent sur un jeu de cartes proche d'un tarot, dont l'existence est ancrée dans l'univers de jeu :

Dans Everway, le jeu de cartes est réellement incorporé dans le récit du jeu, au contraire des systèmes basés sur les dés dans lesquels ces derniers constituent un moyen de résoudre les incertitudes mais ne sont pas présents dans le récit. Dans Everway, les règles expliquent comment les « dieux et déesses ont créé le Jeu de la Destinée comme un guide pour l'humanité » [...] Les trente-six " images sont dessinées suivant un schéma spécifique. Elles détaillent l'évolution de l'âme humaine depuis sa manifestation dans le monde, au travers de différentes vies ou conditions, jusqu'à son inévitable perfection finale ». [...] En incorporant le jeu de cartes dans le métarécit du jeu, les mécanismes d'Everway s'insinuent dans la narration du jeu de rôle (MacKay, 2001, p.42-43, traduction personnelle).

Les innovations esthétiques dans Everway rapprochent le système formel de l'univers de jeu. Tweet reprend des conceptions anciennes du monde et de l'homme pour en chercher des traductions logico-mathématiques. Les personnages sont décrits par une quantification et une combinatoire des humeurs (bile jaune, bile noire, sang...) et des éléments (feu, terre...) proches des anthropologies médiévales. Ceci évite d'appliquer un vocabulaire technique à un univers mystique. La convergence d'expression du simulant et du simulé se retrouve aujourd'hui dans de nombreux jeux de rôle.

\section{Jeux vidéo : deux esthétiques du détail}

7 Le cas des jeux vidéo est, d'un point de vue simiotique et esthétique, diamétralement opposé aux deux précédents. Inutile d'apprendre la règle du jeu, puisque les opérations de simulation sont prises en charge par la machine. Eternal Darkness: Sanity's Requiem (Silicon Knights, 2002) inclut par exemple un système de santé mentale comparable à celui de L'Appel de Cthulhu, mais celui-ci ne se révèle au joueur qu'au fil de son aventure cauchemardesque.

Contrairement aux règles explicites des jeux de plateau et des jeux de rôle, les moteurs physiques et graphiques des jeux vidéo ne dévoilent le plus souvent leurs propriétés qu'à l'usage, par les résultats qu'ils produisent. Leur potentiel esthétique est là : la simulation se distingue de la représentation et du récit par le fait qu'elle engendre des représentations et, a posteriori, des récits propres à l'usage qu'en fait le joueur (Frasca, 2001b). De plus, un jeu vidéo ne se juge pas à l'aune de la seule partie jouée, mais au regard de l'éventail des parties jouables. Le critique en obtient un aperçu en multipliant 
les choix tactiques et les comparaisons d'un système à l'autre. Plus encore que le lecteur ou le spectateur d'une fiction mimétique, le joueur est donc dans une large mesure créateur de son expérience esthétique, au sens où son aptitude à mettre à l'épreuve les limites du simulateur conditionne l'appréciation qu'il en aura.

Les deux piliers du jugement simiotique sur les jeux vidéo sont l'adéquation technique entre simulant et simulé et la mise en relief des potentialités du simulateur. Par exemple, si le jeu permet de simuler la conduite d'un véhicule avec un pneu crevé, il faut que les "sensations de conduite" paraissent correspondre à cette situation (adéquation technique) et que l'aventure amène le personnage à fuir des adversaires en roulant sur la jante (exploitation scénaristique de cette potentialité du simulateur). Malgré des failles unanimement relevées par les critiques (linéarité, personnage sans charisme, adversaires prévisibles), Half Life 2 (Valve 2004) a été couvert de récompenses pour son exploitation particulièrement riche du moteur Havok :

Les puzzles par exemple feront souvent appel au moteur physique du jeu, en nous demandant d'alourdir une plate-forme afin de déclencher une réaction en chaîne. Si le concept est banal, la forme est au moins originale. C'est ce que l'on se dit lorsqu'on doit retirer les cales d'un blindé en charge sur un poteau électrique afin de le faire rouler et ainsi d'abattre ledit poteau (Dinowan, 2008).

La mise en relief des potentialités du simulateur peut être obtenue par divers procédés. Il peut s'agir d'un problème qui se résout par différentes tactiques sollicitant plusieurs mécanismes du moteur (tir, magie, piratage, combat rapproché, infiltration) ou d'un enchaînement scénarisé de situations, d'armes, de véhicules qui renouvelle l'expérience tout en soulignant les qualités du moteur. Dans Half Life 2, on manie un équipement conçu pour exploiter les qualités du moteur : une carabine anti-gravité qui attire et projette des objets. Elle permet d'improviser toutes sortes de manœuvres : construire une barricade ou un escalier avec des caisses, faire s'effondrer l'échafaudage d'où tirent les adversaires, projeter sur eux une voiture ou un tonneau d'explosifs. Si Havok est considéré comme l'un des meilleurs moteurs physiques par rapport aux capacités des machines disponibles en 2004 (flanc axiomatique), si les graphismes, les musiques et les sons sont jugés réussis (flanc mimétique), c'est surtout la qualité « simiotique » du jeu qui le singularise, car tout est fait pour offrir au joueur une variété d'expériences émotionnelles et tactiques :

Les scènes d'anthologie s'enchaînent, qu'il s'agisse de la séquence en hydroglisseur ou à bord du buggy entrecoupées de haltes afin de débloquer le passage en faisant flotter un tremplin ou en replaçant une batterie sur un contacteur. Viennent aussi les combats contre des hélicos de la Milice et d'autres. Valve sait continuellement renouveler les plaisirs et nous happer dans son univers. Ici on se la jouera James Bond dans les marais ou les canaux en hydroglisseur, là on prendra d'assaut une base ennemie en compagnie de créatures mutantes maintenues sous notre contrôle à l'aide de phéromones, juste après qu'elles aient elles-mêmes failli nous écharper, là on ira lutter en ville contre une horde d'araignées géantes, peu après avoir attaqué une prison, etc. (Dinowan, 2008.).

51 L'appréciation simiotique porte de plus en plus sur ces moteurs qui équipent indifféremment des jeux très variés. Ces simulateurs sont conçus pour recréer des processus environnementaux indépendamment de l'enjeu et de l'intrigue, donc leur dimension esthétique parait peu pertinente aux sciences du jeu. Plus intéressantes sont les trouvailles qui enrichissent l'expérience d'un jeu en particulier par des aménagements locaux du moteur ou de l'environnement.

52 La première esthétique du détail est adressée au joueur en tant que spectateur/ explorateur : il s'agit d'orner la diégèse d'éléments inutiles au jeu, mais qui néanmoins 
réagissent à l'action (au contraire des simples ornements graphiques et sonores d'ambiance). Les pigeons dans la série Assassin's Creed (Ubisoft, 2007-) illustrent bien cette démarche: ils s'intègrent dans les paysages urbains et s'envolent à l'approche du personnage, mais ne servent à rien. Il s'agit d'une version simiotique de l'« effet de réel » théorisé par Roland Barthes, «fondement de ce vraisemblable inavoué qui forme l'esthétique de toutes les œuvres courantes de la modernité » (Barthes, 1968, p. 88) : le détail superflu, qui fait partie de l'œuvre mais échappe à sa structure, renforce l'atmosphère de la diégèse et la possibilité d'immersion en son sein.

La deuxième manifestation esthétique du détail est plus clairement liée au gameplay. Examinons deux trouvailles dans le domaine des jeux de tir : le bullet time et la killcam. La première est une technique venue du cinéma, popularisée notamment par Matrix en 1999. Elle consiste à suivre au ralenti le trajet d'un objet, le plus souvent une balle. Max Payne (Rockstar, 2001) propose cette possibilité de ralentir ponctuellement le temps lors d'un tir, créant à la fois un enrichissement du jeu et une référence au cinéma d'action. La killcam est un procédé voisin, qui permet de voir la mort d'un personnage du point de vue de son tueur ou de suivre une balle (une flèche dans Skyrim, Bethesda Game Studios, 2011) sur toute sa trajectoire, au ralenti. C'est une manière de tirer parti du fonctionnement du simulateur pour créer un effet audiovisuel saisissant, parfois critiqué comme répétitif et contre-immersif. Mais la killcam peut, comme le bullet time, être exploitée à des fins proprement ludiques, c'est-à-dire pour aider le joueur à s'améliorer. C'est le cas dans Sniper Elite V2 (Rebellion Developments, 2012), dont le simulateur incorpore des contraintes du tir de précision souvent négligées, comme la gravité et le vent. La killcam montre non seulement l'impact de la balle, mais aussi les os et les organes internes de la victime, ce qui devient utile pour infliger différents types de dégâts. On associe donc, via un aménagement du simulateur, un effet mimétique original et un apprentissage nouveau pour le joueur.

54 L'esthétique des simulateurs vidéoludiques oscille dont entre une exigence générale de performance soumise à la pression technologique et la valorisation d'entorses ponctuelles au fonctionnement audiovisuel du moteur pour permettre un enrichissement des visualisations et des situations de jeu.

\section{Conclusion}

Savoir si les jeux sont ou non des arts me semble une manière maladroite de poser une bonne question. Il est aujourd'hui évident que l'édition ludique requiert de nombreux artistes pour enrichir le rendu visuel, tactile ou sonore d'un jeu, et que l'on peut donc exposer des jeux de rôle, des jeux de plateau ou des jeux vidéo dans des musées comme autant de « beaux objets ». La cinématique d'ouverture d'un jeu vidéo ou la boîte d'un jeu de société ont tout à gagner à ce renfort artistique. Mais il me semble que le sujet esthétique central pour les sciences du jeu dépasse cette dimension «ornementale » et concerne davantage l'apport spécifique des jeux à nos conceptions du beau.

Il s'agit de mieux comprendre les effets esthétiques liés au jeu en tant qu'expérience, ceux qui ne sont pas transposables à l'adaptation d'un jeu en roman, en bande dessinée ou en film. À ce titre, j'ai tenté de définir des adjectifs souvent employés autour des jeux de tables, comme "subtil », " épuré » ou " élégant ", mais aussi de cerner certains ressorts plus discrets de l'expérience ludique, comme l'activité silencieuse des problémistes et des 
solutionnistes. Nombre d'auteurs de jeux parlent d'un plaisir propre au game design le plus sobre, celui où l'on ne manipule que des croquis, des cubes de bois, des opérateurs logiques. C'est sur cette voie que j'ai tenté, en suivant Nabokov et Smullyan, de mettre en relief l'esthétique propre du jeu, depuis la démarche exploratoire d'un auteur jusqu'aux différentes utilisations du produit final.

\section{BIBLIOGRAPHIE}

BARONI R. et MARTI M. (2014), « De l'interactivité du récit au récit interactif », Cahiers de narratologie, 27 (http://narratologie.revues.org).

BARTHES R. (1968), « L'effet de réel », Communications, 11, pp. 84-89.

CAILLOIS R. (1967), Les jeux et les hommes : le masque et le vertige [1958], Paris, Gallimard.

CAÏRA O. (2011), Définir la fiction : du roman au jeu d'échecs, Paris, Éditions de l'EHESS.

CHÉRON A. (1971), Les échecs artistiques, Paris, Payot.

CLÉRO J.-P. (2004), Les raisons de la fiction : les philosophes et les mathématiques, Paris, Armand Colin. DINOWAN (2008), « Test : Half Life 2 », www.jeuxvideo.com (consulté le 30 mai 2016).

FRASCA G. (2001a), Videogames of the Oppressed: Videogames as a Means for Critical Thinking and Debate (Master of Information Design and Technology), Georgia Institute of Technology.

FRASCA G. (2001b), « Simulation 101 : Simulation versus Representation », (www.ludology.org)

GOFFMAN E. (1991), Les cadres de l'expérience [1974], Paris, Éditions de Minuit.

HASAN-ROKEM G. et SHULMAN D. (1996), Untying the Knot : Riddles and Other Enigmatic Modes, Oxford, Oxford University Press.

HOFSTADTER D. (2000), Gödel, Escher, Bach : les brins d'une guirlande éternelle [1979] Paris, Dunod.

KANT E. (1989), Critique de la faculté de juger [1790], Paris, Gallimard.

LAUTIER J. (2002), « Préface », Larousse du jeu d'échecs, Paris, Larousse, pp 304-305.

MACKAY D. (2001), The Fantasy Role-Playing Game: A New Performing Art, Jefferson NC, McFarland \& Co.

NABOKOV V. (1991), Autres rivages [1951], Paris, Gallimard.

RYAN M.-L. (2001), Narrative as Virtual Reality: Immersion and Interactivity in Literature and Electronic Media, Baltimore, The Johns Hopkins University Press.

RYAN M.-L. (2006), Avatars of Story, Minneapolis, Minnesota University Press.

SCHAEFFER J.-M. (1999), Pourquoi la fiction ?, Paris, Éditions du Seuil.

SMULLYAN R. (1983), Mystères sur échiquier avec Sherlock Holmes [1979], Paris, Dunod. 


\section{NOTES}

1. Je désigne ainsi la vaste famille des énoncés mathématiques qui présentent une situation fictionnelle («Un robinet remplit une baignoire au rythme de 2 litres par minute », «Un train roule à $250 \mathrm{~km} / \mathrm{h}$ entre les villes $\mathrm{A}$ et $\mathrm{B} »)$ afin de favoriser la compréhension des calculs attendus.

\section{RÉSUMÉS}

Les jeux occupent aujourd'hui une place centrale dans la palette des fictions. Si les jeux abstraits constituent un pôle autonome d'expression logico-mathématique, les jeux de simulation sont en revanche créés par association d'un thème et d'un système, ce qui les rend à la fois mimétiques et axiomatiques. On étudie ici différentes manifestations de plaisir esthétique liées à l'expérience des jeux, indépendamment de leur ornementation visuelle ou sonore. Pour les jeux abstraits, on identifie trois motifs: le sublime qui reflète notre incapacité à embrasser l'immensité d'une combinatoire, le subtil qui se révèle lors de la résolution d'énigmes originales ou difficiles, et le merveilleux qui découle de la découverte de propriétés inattendues du système. Les jeux de simulation sont étudiés en deux temps : les jeux de plateau et de rôle dont le système fonctionne comme une épure, et les jeux vidéo qui associent performance générale du moteur et création d'écarts esthétiques par le détail.

Games hold a central position in the range of contemporary fictions. While abstract games form an autonomous cluster of logical-mathematical expression, simulation games are designed by associating a theme and a system, which makes them both mimetic and axiomatic. This paper studies several expressions of aesthetic pleasure in relation to the experience of games, regardless of their image or sound quality. In the case of abstract games, three forms are identified: "sublime" reflects our inability to grasp the countless combinations of a system, "subtle" is expressed when handling original or difficult puzzles, "marvellous" is prompted by the discovery of unexpected properties within the rules. Simulation games are examined in two sections. In the case of board and roleplaying games, the simulation system is stylised so that users can handle it. On the contrary, computer games are based on complex systems, but designers locally modify the simulation engine or the environment to create aesthetic details.

\section{INDEX}

Keywords : aesthetics, board game, Chess, computer game, fiction, game, roleplaying game, sublime

Mots-clés : Échecs, esthétique, fiction, jeu, jeu vidéo, jeu de plateau, jeux de rôle, sublime 
AUTEUR

OLIVIER CAÏRA

Université d'Evry 